\title{
Breakdown of the X-Ray Resonant Magnetic Scattering Signal during Intense Pulses of Extreme Ultraviolet Free-Electron-Laser Radiation
}

\author{
L. Müller, ${ }^{1, *}$ C. Gutt, ${ }^{1,2}$ B. Pfau, ${ }^{3}$ S. Schaffert, ${ }^{3}$ J. Geilhufe, ${ }^{4}$ F. Büttner, ${ }^{3}$ J. Mohanty, ${ }^{3}$ S. Flewett, ${ }^{3}$ R. Treusch, ${ }^{1}$ \\ S. Düsterer, ${ }^{1}$ H. Redlin, ${ }^{1}$ A. Al-Shemmary, ${ }^{1}$ M. Hille, ${ }^{5}$ A. Kobs, ${ }^{5}$ R. Frömter ${ }^{5}$ H. P. Oepen, ${ }^{5}$ B. Ziaja,${ }^{1,2,6,7}$ N. Medvedev, ${ }^{1,6}$ \\ S.-K. Son, ${ }^{1,6}$ R. Thiele, ${ }^{1,6}$ R. Santra, ${ }^{1,2,6,8}$ B. Vodungbo, ${ }^{9}$ J. Lüning, ${ }^{9}$ S. Eisebitt,,${ }^{3,4}$ and G. Grübel ${ }^{1,2}$ \\ ${ }^{1}$ Deutsches Elektronen-Synchrotron (DESY), Notkestraße 85, 22607 Hamburg, Germany \\ ${ }^{2}$ The Hamburg Centre for Ultrafast Imaging, Luruper Chaussee 149, 22761 Hamburg, Germany \\ ${ }^{3}$ Institut für Optik und Atomare Physik, TU Berlin, 10623 Berlin, Germany \\ ${ }^{4}$ Helmholtz-Zentrum Berlin für Materialien und Energie GmbH, 14109 Berlin, Germany \\ ${ }^{5}$ Institut für Angewandte Physik, Universität Hamburg, 20355 Hamburg, Germany \\ ${ }^{6}$ Center for Free-Electron Laser Science, DESY, 22607 Hamburg, Germany \\ ${ }^{7}$ Institute of Nuclear Physics, Polish Academy of Sciences, Radzikowskiego 152, 31-342 Krakow, Poland \\ ${ }^{8}$ I. Institut für Theoretische Physik, Universität Hamburg, 20355 Hamburg, Germany \\ ${ }^{9}$ Laboratoire de Chimie Physique Matière et Rayonnement-CNRS UMR 7614, Université Pierre et Marie Curie, 75005 Paris, France
} (Received 11 October 2012; published 7 June 2013)

\begin{abstract}
We present results of single-shot resonant magnetic scattering experiments of $\mathrm{Co} / \mathrm{Pt}$ multilayer systems using $100 \mathrm{fs}$ long ultraintense pulses from an extreme ultraviolet (XUV) free-electron laser. An x-rayinduced breakdown of the resonant magnetic scattering channel during the pulse duration is observed at fluences of $5 \mathrm{~J} / \mathrm{cm}^{2}$. Simultaneously, the speckle contrast of the high-fluence scattering pattern is significantly reduced. We performed simulations of the nonequilibrium evolution of the $\mathrm{Co} / \mathrm{Pt}$ multilayer system during the XUV pulse duration. We find that the electronic state of the sample is strongly perturbed during the first few femtoseconds of exposure leading to an ultrafast quenching of the resonant magnetic scattering mechanism.
\end{abstract}

DOI: 10.1103/PhysRevLett.110.234801

PACS numbers: 41.60.Cr, 75.70.-i, 78.70.Ck

The use of elastic x-ray scattering for studying electronic and magnetic structures of matter is based on the premise that the scattered $x$ rays provide information on an as-prepared state. The new free-electron-laser (FEL) sources of ultraintense and ultrashort pulses of extreme ultraviolet (XUV) and $\mathrm{x}$-ray radiation challenge this premise. Here, we present the results of a resonant magnetic scattering experiment at the FEL source FLASH using ultraintense XUV pulses $\left(5 \mathrm{~J} / \mathrm{cm}^{2}\right)$ tuned to the dichroic transition of cobalt at $20.8 \mathrm{~nm}$ ( $M$ edge). At this fluence we observe an intensity-induced reduction of the x-ray resonant magnetic scattering cross section together with a reduction in speckle contrast. With the help of a microscopic atomic model, we follow the nonequilibrium evolution of the electronic structure during the XUV-FEL pulse duration. We find that the excitation generates already during the first few femtoseconds a strongly perturbed electronic system in which the resonant magnetic scattering mechanism is quenched.

Resonant magnetic $\mathrm{x}$-ray scattering has become an invaluable tool to determine magnetic properties of solids on nanometer length scales. The technique has been developed to such a fine art that today, e.g., orbital and spin magnetic moments can be separately determined on femtosecond time scales using femtoslicing synchrotron sources [1-4]. XUV [5] and x-ray FEL sources, however, allow us to take single-pulse snapshots of magnetic domain configurations [6] with the perspective to study transient and highly excited spin states on femtosecond time scales and with nanometer spatial resolution [7]. Lensless imaging techniques [8] make use of the high-coherence properties of the FEL beam to record magnetic holograms within a single sub-100 fs FEL pulse [9].

The resonant magnetic scattering process is element specific and sensitive to the magnetic moments through the same x-ray magnetic circular dichroism effect as exploited in absorption spectroscopy [10,11]. However, when tuning the photon energy to an absorption edge, the radiation damage is also strongly enhanced leading to a trade-off between magnetic scattering and damage to the sample. Recent experiments reporting on x-ray-induced enhanced transmission in aluminum [12] and neon [13] demonstrated the effect of transient electronic states excited by intense femtosecond $\mathrm{x}$-ray pulses on the absorption properties of the samples. Diffraction experiments using nanocrystals showed that the onset of ion movement on time scales of $70 \mathrm{fs}$ and longer terminates the Bragg diffraction condition [14]. Resonant magnetic diffraction probes a specific quantum transition within the electronic system and thus damage can manifest itself even faster than 70 fs.

Resonant magnetic scattering at the $\mathrm{Co} L_{3}$ edges $(1.6 \mathrm{~nm})[9,15]$ and single-pulse scattering at the Co $M_{3}$ edges $(20.8 \mathrm{~nm})$ [6] from $\mathrm{Co} / \mathrm{Pt}$ magnetic multilayer 

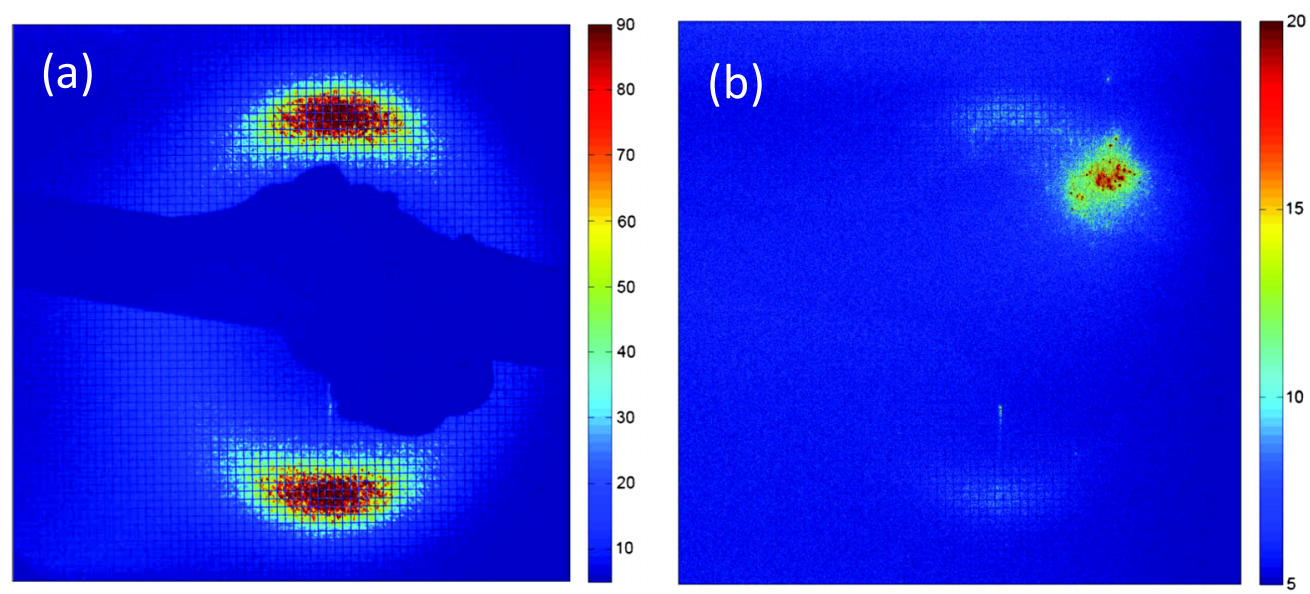

FIG. 1 (color online). Detector images showing the resonant magnetic scattering signal from a Co/Pt magnetic multilayer film in which the magnetic domains have been partially aligned beforehand. The FEL has been tuned to the magnetodichroic transition of cobalt at $20.8 \mathrm{~nm}$. (a) The sum of 1000 low-fluence images with a total flux of $7.5 \mathrm{~J} / \mathrm{cm}^{2}$. (b) Single-shot image using a high incident $\mathrm{x}$-ray fluence of $5 \mathrm{~J} / \mathrm{cm}^{2}$. In both cases the color bar indicates the total number of scattered photons. The parasitic signal in the right-hand image originates from the explosion of the sample and is different in each single shot. The regular grid structure is due to a nickel-mesh stabilized aluminum filter protecting the camera from any visible light.

samples [16,17] showed that FEL fluences of up to $2-4 \mathrm{~mJ} / \mathrm{cm}^{2}$ allow recording single-shot magnetic diffraction patterns without changing the magnetic properties of the sample. Fluences above $9 \mathrm{~mJ} / \mathrm{cm}^{2}$, on the other hand, lead to a heat-induced altering of the domain structure which occurs on picosecond to nanosecond time scales, much slower than the femtosecond pulse duration. Finally, fluences of $20 \mathrm{~mJ} / \mathrm{cm}^{2}$ lead to the destruction of the magnetic multilayer sample with the magnetic scattering signal still intact-that is, the destruction process is too slow to leave a trace in the femtosecond diffraction snapshot. Here, we have used pulse energies of around $20 \mu \mathrm{J}$ and a beam size of $20 \mu \mathrm{m}$ (FWHM) at the sample position yielding $\mathrm{x}$-ray fluences of the order of $5 \mathrm{~J} / \mathrm{cm}^{2}$ within an expected pulse duration of $100 \mathrm{fs}$. The photon energy was set to $59.6 \mathrm{eV}$ corresponding to a wavelength of $20.8 \mathrm{~nm}$ to match the Co $M_{3}$ resonance. FEL pulse intensities were measured with a gas monitor [18] and the experiment was carried out in the chamber described in [19].

In Fig. 1 we show images of the magnetic scattering signal of a $\mathrm{Co} / \mathrm{Pt}$ multilayer film recorded with two different FEL fluences. The scattering pattern in Fig. 1(a) results from summing up the scattering signals from $1000 \mathrm{FEL}$ pulses (repetition rate $10 \mathrm{~Hz}$ ) in one exposure of the camera. The average incident photon flux of these pulses was $7.5 \mathrm{~mJ} / \mathrm{cm}^{2}$. A diffraction pattern with two lobes of intensity is observed which points towards the absence of heat-load effects, as a state of linearly aligned magnetic domains would become disordered towards a $2 d$ labyrinth state upon heating, such that the scattering image would evolve into a ring. Magnetic speckles are visible, and by plotting the probability density of the speckle intensity (Fig. 2) we obtain $M=24$ contributing modes, which corresponds to a speckle contrast of $1 / \sqrt{M}=20 \%$ [20].
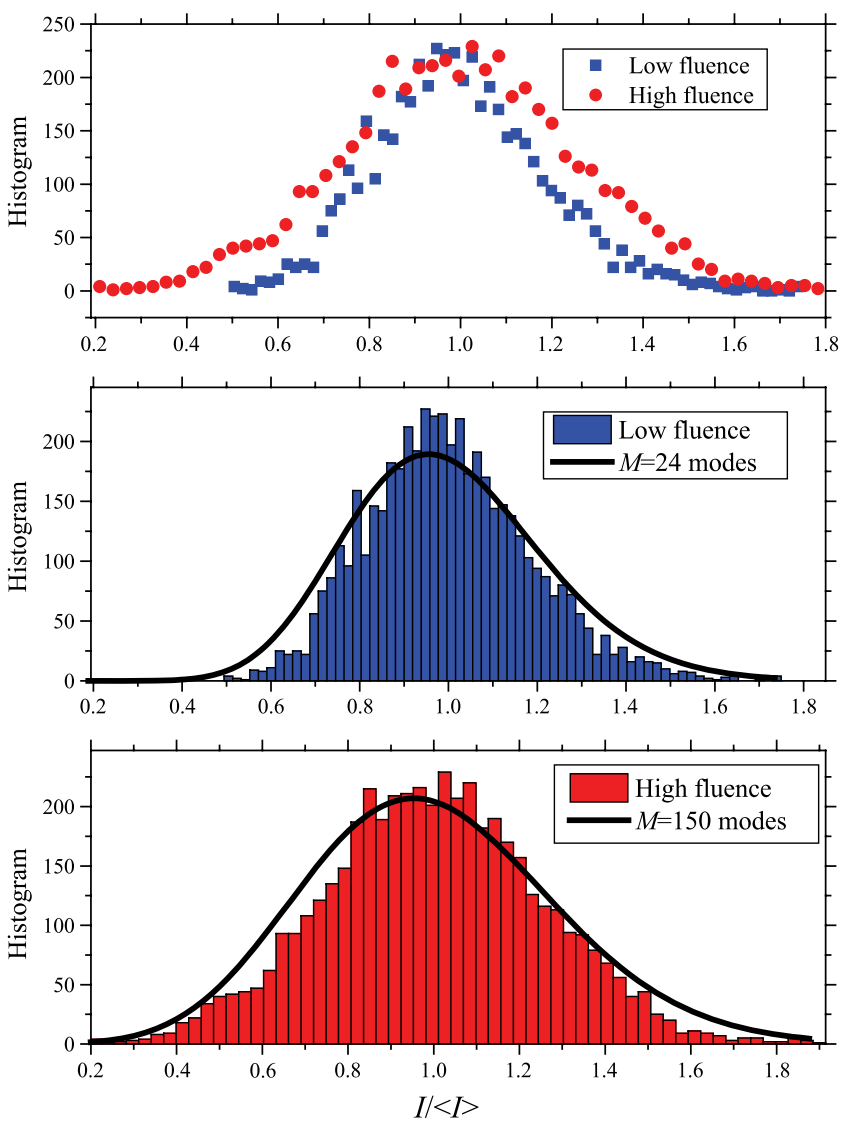

FIG. 2 (color online). Probability density functions of the speckle intensity for the low- (blue) and high-fluence (red) speckle pattern from Fig. 1. The black solid lines are fits with Eq. (S1) (in the Supplemental Material) giving the number of modes $M$ contributing to the speckle image (see Supplemental Material [21]). 
The observation of such a high value of speckle contrast in the averaged image implies that the domain pattern is not changing during the exposure and confirms that the diffraction pattern represents an as-prepared magnetic stripe-domain state.

Figure 1(b) shows the magnetic scattering from a single shot with an intensity of $5 \mathrm{~J} / \mathrm{cm}^{2}$ recorded on the same sample as the multishot low-fluence exposure shown in Fig. 1(a). Two differences are evident: a strongly reduced scattering intensity of the previously intense scattering lobes and the appearance of an additional signal on the detector. The latter signal is parasitic in that it varies in shape, position, and intensity from shot to shot. We attribute this signal to the Coulomb explosion of the sample. It can be excluded that this signal originates from a scattering process due to its shape and lack of point symmetry; therefore, the signal's position on the detector cannot be related to a $Q$ value in contrast to the scattering signal from the magnetic domains. The observation of interest in the context of this Letter, however, is the strong reduction of the resonant magnetic scattering intensity relative to the low-fluence case. In Fig. 3 we display the radially averaged magnetic scattering intensity versus the momentum transfer $Q$ of the magnetic domains with both curves normalized to the incident photon flux. The high-fluence measurement displays a reduction of the resonant magnetic scattering intensity by a factor of $20 \pm 5$. Altogether, four pairs of multi- and single-shot images have been taken in such a way and all show the same behavior with respect to the diminishing of the scattering signal for the high-fluence measurements (see Supplemental Material [21]).

We attribute the loss in scattering intensity to a reduction in the efficiency of the resonant magnetic scattering process. While we note that at high fluences processes such as stimulated emission and recombination can become important

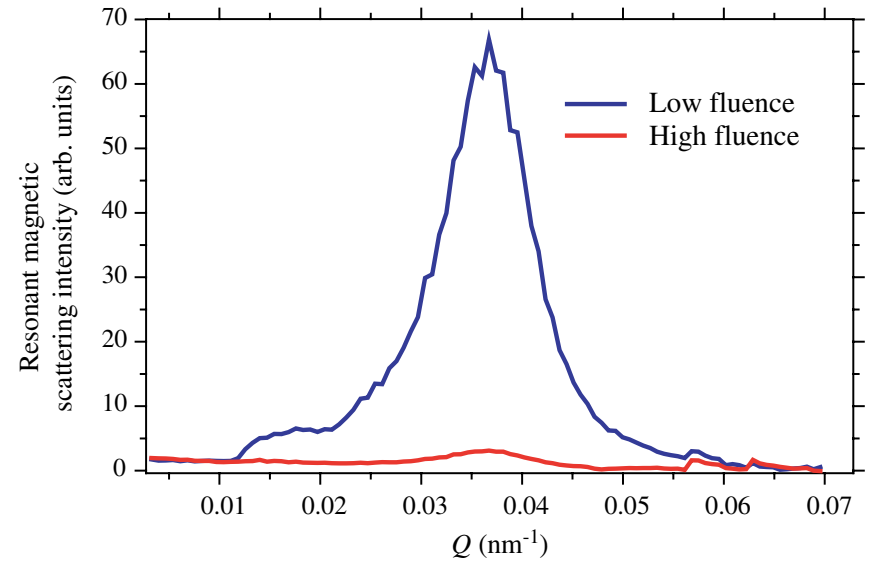

FIG. 3 (color online). The azimuthally integrated resonant magnetic scattering signals from Fig. 1 normalized to the incident photon flux. The blue line represents the low-fluence measurement with $7.5 \mathrm{~mJ} / \mathrm{cm}^{2}$ and the red line the high-fluence measurement with $5 \mathrm{~J} / \mathrm{cm}^{2}$.
[22], we propose that the intensity reduction observed here is to a large extent due to $\mathrm{x}$-ray absorption which leads to significant changes in the material's electronic structure. Of these, the photoionization of weakly bound $3 d$ and $4 s$ electrons are relevant. As a consequence, the binding energy of the remaining $3 p$ electrons increases and the energy of the incident photons is no more suitable to induce resonant transitions involving these shifted energy levels.

To substantiate this idea we have performed simulations of the nonequilibrium evolution of the $\mathrm{Co} / \mathrm{Pt}$ multilayer sample which yield quantitative insights into the ultrafast electronic processes occurring during the intense FEL irradiation (for details see Supplemental Material [21]). The calculations allow following the complex nonequilibrium evolution of the electronic and atomic system starting from the neutral state through all stages of the progressing ionization and sample damage up to a possible explosion occurring at sufficiently high radiation doses [23]. We considered two pulse-duration values of 30 and $100 \mathrm{fs}$ with fixed pulse fluence levels but restrict our discussion in the following to the results of the $100 \mathrm{fs}$ calculation.

The simulations reveal that the movement of the atoms during the XUV pulse duration of $100 \mathrm{fs}$ is small with an average atomic displacement of only about $0.01 \mathrm{~nm}$. Larger displacements due to the Coulomb explosion occur on longer time scales after the FEL pulse. Thus, structural changes are negligible for the scattering process, and we can concentrate on the electronic system only. In the case of low fluence of $7.5 \mathrm{~mJ} / \mathrm{cm}^{2}$ only minor changes are induced to the electronic system: the average ionization degree per atom is $\approx 0.01$ and only Co +1 states appear within the sample [Fig. 4(a)]. The few excited photoelectrons rapidly lose their energy in inelastic collisions, so the kinetic temperature of the electron cloud decreases fast, without electron-electron thermalization, as the density of the free electrons is too low (Fig. 5). In the high-fluence level case of $5 \mathrm{~J} / \mathrm{cm}^{2}$, the electronic system and the average ionization degree per atom change dramatically after a few up to tens of femtoseconds. For the simulated exposures with 30-100 fs long FEL pulses, the atomic cobalt evolves to a Co $1+$ state after $2-10$ fs, to Co $2+$ after 5-20 fs, and then to Co 3+ state after 6-30 fs [Fig. 4(b)]. The average ionization degree is already close to 1 after 4-10 fs. Because of the high density, the photoelectrons thermalize quickly (after 5-15 fs) and reach a temperature of $\approx 22 \mathrm{eV}$ (Fig. 5). Note that all simulations are based on a $\mathrm{Co} / \mathrm{Pt}$ multilayer system, thus also taking into account the free electrons generated in the platinum. The resulting ionization degree of platinum is comparable with the one of cobalt (see Supplemental Material [21]).

Using a dedicated code for Hartree-Fock-Slater model calculations (ХАTOM package [24]) extended to include the effects of the dense free electron environment on the atomic potential of the central atom (extended XATOM package [25]), we have estimated the energy of the 

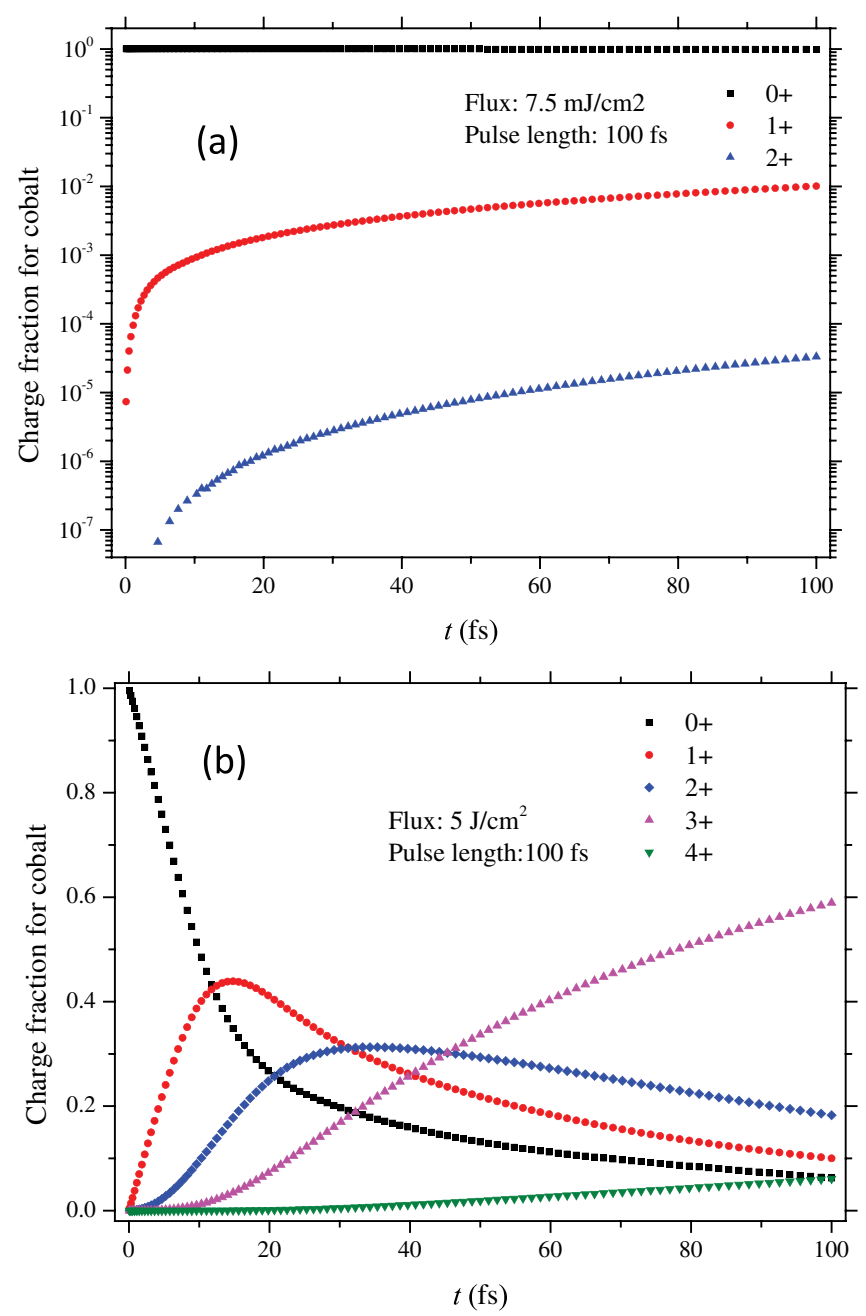

FIG. 4 (color online). Simulation results of the evolution of the $\mathrm{Co} / \mathrm{Pt}$ multilayer system during the exposure to a $100 \mathrm{fs}$ long FEL pulse. Fraction of differently charged cobalt ions at (a) low and (b) high FEL pulse fluences.

resonant transition in (i) isolated cobalt atoms and ions and (ii) when screened by the cloud of free electrons. In these calculations the resonant transition of isolated unscreened cobalt atoms occurs at a photon energy of $E \approx 59 \mathrm{eV}$. The photoionization in our model will most probably occur in the $3 d$ shell resulting in a Co $1+$ ion with resonant transition energy of $\approx 60 \mathrm{eV}$. This is a shift of $\approx 1 \mathrm{eV}$ towards higher energies, while the photon bandwidth is $0.6 \mathrm{eV}$; i.e., the photon energy is now slightly out of resonance. For Co $2+$ the relative shift increases to $\approx 1.5 \mathrm{eV}$ and for Co $3+$ to $\approx 2 \mathrm{eV}$. Accounting for the screening effect we obtain relative shifts of 1.8 and $2.1 \mathrm{eV}$, respectively. See also Ref. [26]. We conclude that in both cases the predicted shifts of the transition energy within the created ions will result in a reduction of the scattering intensity with the FEL staying tuned to the cobalt $0+$ bulk transition energy. Using the energy dependence of the Kramers-Heisenberg relation, we calculate a factor of at least 5 in intensity reduction. This is less than observed, which we attribute

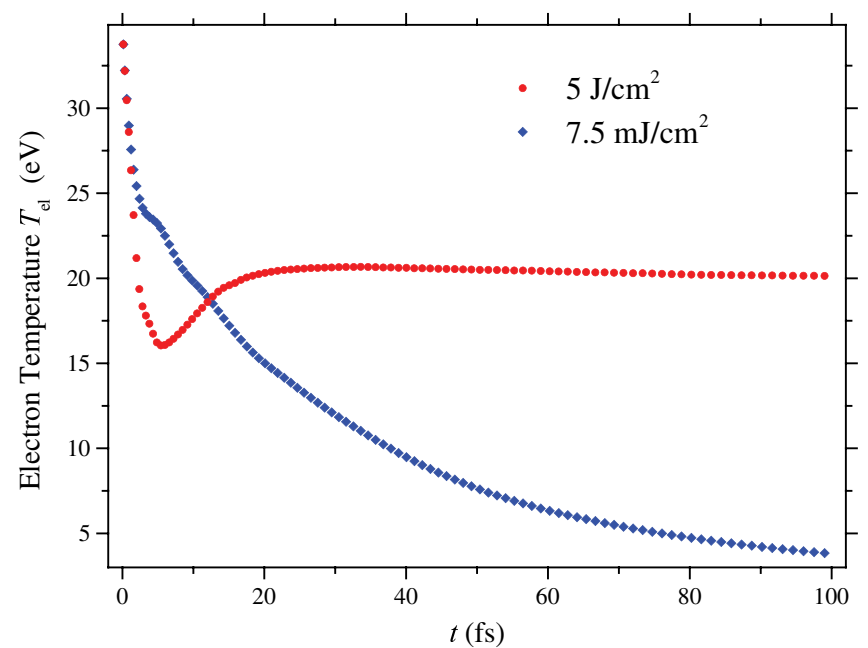

FIG. 5 (color online). Simulation results of the kinetic temperature evolution of the free electrons of a $\mathrm{Co} / \mathrm{Pt}$ multilayer system during the exposure to a $100 \mathrm{fs}$ long FEL pulse at the fluences used in the experiment.

to shortcomings of our simplified atomistic model of cobalt (e.g., neglecting resonant excitations during the beginning of the pulse) and other channels contributing to the reduction of the resonant scattering process.

Since the details of the evolution of the sample's electronic state depend on the fluence, one has to conclude that a laterally inhomogeneous pulse fluence results in a lateral structuring of the electronic state within the sample. An inhomogeneous profile of an intense FEL pulse, such as a simple Gaussian shape, will therefore yield a spatially inhomogeneous bleaching of the scattering mechanism. This in turn leads to a superposition of slightly different speckle patterns during the exposure on the detector giving rise to a lower speckle contrast in the summed image. This conclusion is supported by a more detailed analysis of the single-shot speckle patterns like the one shown in Fig. 1(b). The speckle intensity distribution shown in Fig. 2 reveals a significant broadening in the high-fluence case, which can be characterized by an increase of the effective number of modes from 24 to 150 . This implies a significant reduction in speckle contrast.

We note that our result implies the existence of a photonflux limitation for ultrafast resonant magnetic scattering. $\mathrm{X}$-ray fluences of $5 \mathrm{~J} / \mathrm{cm}^{2}$ are so high that the resonant scattering mechanism is quenched during the $\mathrm{x}$-ray exposure. The simulations verify that only the first few femtoseconds of exposure probe the as-prepared electronic and spin system. Consequently, the availability of even shorter FEL pulses is essential to outrun the electronic damage.

This research was carried out at the light source FLASH at DESY, a member of the Helmholtz Association (HGF). We would like to thank the scientific and technical team at FLASH for their support during the experiment. Financial support by the DFG within SFB 925 is gratefully acknowledged. S. E. and S. S. acknowledge support from 
the BMBF under Contract No. 05K10KTB/FSP-301. The University of Hamburg group acknowledges support within SFB668 and from BMBF under Contract No. 05K10GU4/FSP-301. B. V. and J. L. acknowledge support from the CNRS through the PEPS SASELEX and from the French ANR via the FEMTO-X-MAG project. This work has been supported by the excellence cluster "The Hamburg Centre for Ultrafast Imaging-Structure, Dynamics, and Control of Matter at the Atomic Scale" of the Deutsche Forschungsgemeinschaft. R. S., S. E., and G. G. acknowledge support in the Helmholtz Virtual Institute "Dynamic Pathways in Multidimensional Landscapes" (VH-VI-419).

*Leonard.Mueller@desy.de

[1] J. Stöhr and H.C. Siegmann, Magnetism, from Fundamentals to Nanoscale Dynamics (Springer, Berlin, 2006).

[2] C. Stamm, T. Kachel, N. Pontius, R. Mitzner, T. Quast, K. Holldack, S. Khan, C. Lupulescu, E. F. Aziz, M. Wietstruk et al., Nat. Mater. 6, 740 (2007).

[3] C. Boeglin, E. Beaurepaire, V. Halté, V. López-Flores, C. Stamm, N. Pontius, H. A. Dürr, and J.-Y. Bigot, Nature (London) 465, 458 (2010).

[4] A. Tanaka, C.F. Chang, M. Buchholz, C. Trabant, E. Schierle, J. Schlappa, D. Schmitz, H. Ott, P. Metcalf, L. H. Tjeng et al., Phys. Rev. Lett. 108, 227203 (2012).

[5] W. Ackermann, G. Asova, V. Ayvazyan, A. Azima, N. Baboi, J. Baehr, V. Balandin, B. Beutner, A. Brandt, A. Bolzmann et al., Nat. Photonics 1, 336 (2007).

[6] C. Gutt, S. Streit-Nierobisch, L.-M. Stadler, B. Pfau, C. M. Günther, R. Könnecke, R. Frömter, A. Kobs, D. Stickler, H. P. Oepen et al., Phys. Rev. B 81, 100401(R) (2010).

[7] B. Pfau, S. Schaffert, L. Müller, C. Gutt, A. Al-Shemmary, F. Büttner, R. Delauny, S. Düsterer, S. Flewett, R. Frömter et al., Nat. Commun. 3, 1100 (2012).

[8] S. Eisebitt, J. Lüning, W.F. Schlotter, M. Lörgen, O. Hellwig, W. Eberhard, and J. Stöhr, Nature (London) 432, 885 (2004).

[9] T. Wang, D. Zhu, B. Wu, C. Graves, S. Schaffert, T. Rander, L. Mueller, B. Vodungbo, C. Baumier, D. P. Bernstein et al., Phys. Rev. Lett. 108, 267403 (2012).

[10] J.P. Hannon, G. T. Trammell, M. Blume, and D. Gibbs, Phys. Rev. Lett. 61, 1245 (1988).
[11] J. B. Kortright, S.-K. Kim, G. P. Denbeaux, G. Zeltzer, K. Takano, and E.E. Fullerton, Phys. Rev. B 64, 092401 (2001).

[12] B. Nagler, U. Zastrau, R. R. Fäustlin, S. M. Vinko, T. Whitcher, A. J. Nelson, R. Sobierajski, J. Krzywinski, J. Chalupsky, E. Abreu et al., Nat. Phys. 5, 693 (2009).

[13] L. Young, E. P. Kanter, B. Kraessig, Y. Li, A. M. March, S. T. Pratt, R. Santra, S. H. Southworth, N. Rohringer, L. F. DiMauro et al., Nature (London) 466, 56 (2010).

[14] A. Barty, C. Caleman, A. Aquila, N. Timneanu, L. Lomb, T. A. White, J. Andreasson, D. Arnlund, S. Bajt, T. R. M. Barends et al., Nat. Photonics 6, 35 (2012).

[15] C. Gutt, L. M. Stadler, S. Streit-Nierobisch, A.P. Mancuso, A. Schropp, B. Pfau, C. M. Günther, R. Könnecke, J. Gulden, B. Reime et al., Phys. Rev. B 79, 212406 (2009).

[16] H. Stillrich, C. Menk, R. Froemter, and H. P. Oepen, Proceedings of the 53rd Annual Conference on Magnetism and Magnetic Materials, 2008 (AIP, New York, 2009); [J. Appl. Phys. 105, 07 C308 (2009)].

[17] O. Hellwig, A. Berger, J. B. Kortright, and E. E. Fullerton, J. Magn. Magn. Mater. 319, 13 (2007).

[18] K. Tiedtke, A. Azima, N. von Bargen, L. Bittner, S. Bonfigt, S. Düsterer, B. Faatz, U. Fühling, M. Gensch, C. Gerth et al., New J. Phys. 11, 023029 (2009).

[19] L. Müller, C. Gutt, S. Streit-Nierobisch, M. Walther, S. Schaffert, B. Pfau, J. Geilhufe, F. Büttner, S. Flewett, C. M. Günther et al., Rev. Sci. Instrum. 84, 013906 (2013).

[20] J. W. Goodman, Statistical Optics (John Wiley \& Sons, New York, 2000).

[21] See Supplemental Material at http://link.aps.org/ supplemental/10.1103/PhysRevLett.110.234801 for details on experiment, simulation methods, and further experimental and simulation data.

[22] N. Rohringer, D. Ryan, R. A. London, M. Purvis, F. Albert, J. Dunn, J. D. Bozek, C. Bostedt, A. Graf, R. Hill et al., Nature (London) 481, 488 (2012).

[23] B. Ziaja, A. R. B. de Castro, E. Weckert, and T. Moeller, Eur. Phys. J. D 40, 465 (2006).

[24] S.-K. Son, L. Young, and R. Santra, Phys. Rev. A 83, 033402 (2011).

[25] R. Thiele, S.-K. Son, B. Ziaja, and R. Santra, Phys. Rev. A 86, 033411 (2012).

[26] S. M. Vinko, O. Ciricosta, B. I. Cho, K. Engelhorn, H.-K. Chung, C. R. D. Brown, T. Burian, J. Chalupsky, R. W. Falcone, C. Graves et al., Nature (London) 482, 59 (2012). 